\title{
EFEITO DO MEDIADOR NATURAL SIRINGALDEÍDO NA DESCOLORAÇÃO DE CORANTES POR REAGENTES FENTON
}

\author{
C. S. SANTANA ${ }^{1}$, A. AGUIAR ${ }^{1,2}$ \\ ${ }^{1}$ Universidade Federal de São João del-Rei, Departamento de Química, Biotecnologia e \\ Engenharia de Bioprocessos \\ ${ }^{2}$ Universidade Federal de Itajubá, Instituto de Recursos Naturais \\ E-mail para contato: kciasantana_02@hotmail.com
}

RESUMO - Siringaldeído (3,5-dimetoxi,4-hidroxibenzaldeído) é um fenol oriundo de lignina de plantas. Por ser um agente redutor de íons $\mathrm{Fe}^{3+}$, ele pode mediar a reação de Fenton $\left(\mathrm{Fe}^{2+}+\mathrm{H}_{2} \mathrm{O}_{2} \rightarrow \mathrm{Fe}^{3+}+{ }^{\bullet} \mathrm{OH}+\mathrm{OH}^{-}\right)$por aumentar a degradação de poluentes orgânicos. No presente trabalho quatro diferentes corantes foram descoloridos por reagentes Fenton $\left(\mathrm{H}_{2} \mathrm{O}_{2}, \mathrm{Fe}^{2+}\right.$ ou $\left.\mathrm{Fe}^{3+}\right)$ na presença do siringaldeído. Reações com íons $\mathrm{Fe}^{2+}$ foram mais efetivas para descoloração dos corantes e para isso consumiram mais $\mathrm{H}_{2} \mathrm{O}_{2}$. O efeito próoxidante do siringaldeído foi mais observado para os sistemas reacionais contendo íons $\mathrm{Fe}^{3+}$. Por exemplo, os sistemas $\mathrm{Fe}^{3+} / \mathrm{H}_{2} \mathrm{O}_{2}$ e $\mathrm{Fe}^{3+} / \mathrm{H}_{2} \mathrm{O}_{2} /$ siringaldeído atingiram $31 \%$ e $59 \%$ de descoloração para o corante azul de metileno após 60 minutos de reação, respectivamente. $\mathrm{O}$ uso desse mediador natural pode ser promissor por aumentar a efetividade de reagentes Fenton.

\section{INTRODUÇÃO}

Efluentes da indústria têxtil apresentam potencial poluidor de corpos hídricos, pois possuem geralmente elevado $\mathrm{pH}$, altas demandas química e bioquímica de oxigênio (DQO e DBO, respectivamente), sólidos totais dissolvidos, bem como alta capacidade de difusão no meio aquático. A estrutura aromática e complexa dos corantes presentes em efluentes têxteis é resistente à luz, à atividade biológica e a outras condições ambientais degradativas. Tratamentos convencionais não são efetivos para remediar esse tipo de efluente, havendo a necessidade de testar tecnologias alternativas e eficientes, no que se refere ao controle e à prevenção da poluição, como os processos oxidativos avançados (POAs) (Kaushik e Malik, 2009). Os POAs são de grande interesse para aplicações no tratamento e pré-tratamento de compostos não biodegradáveis em águas e solos contaminados, pois podem converter a matéria orgânica em $\mathrm{CO}_{2}$ e $\mathrm{H}_{2} \mathrm{O}$, ou no caso de pré-tratamentos, tornando-os biodegradáveis. Esses processos baseiam-se na produção de radicais livres, principalmente hidroxila $\left({ }^{\bullet} \mathrm{OH}\right)$, que atacam indistintamente todas as moléculas presentes em solução (Aguiar e Ferraz, 2007; Aguiar et al., 2007).

Um dos POAs mais promissores consiste na reação entre $\mathrm{Fe}^{2+}$ e $\mathrm{H}_{2} \mathrm{O}_{2}$, conforme Equação 1, conhecida como reação de Fenton. Essa reação permite obter elevados níveis de depuração à pressão e temperatura ambientes. $\mathrm{O} \mathrm{Fe}^{3+}$ é mais vantajoso de ser usado, pois é 
mais abundante e tem menor custo. Porém, um inconveniente à utilização de $\mathrm{Fe}^{3+}$ refere-se ao fato de que a reação de Fenton torna-se mais lenta, pois é dependente da reação representada pela Equação 2. $\mathrm{O} \mathrm{Fe}$ gerado nesta reação reagirá com $\mathrm{H}_{2} \mathrm{O}_{2}$ segundo Equação 1 (Aguiar et al., 2007).

$$
\begin{array}{lc}
\mathrm{Fe}^{2+}+\mathrm{H}_{2} \mathrm{O}_{2} \rightarrow \mathrm{Fe}^{3+}+{ }^{-} \mathrm{OH}+{ }^{\bullet} \mathrm{OH} & k=76 \mathrm{~L} \mathrm{~mol}^{-1} \mathrm{~s}^{-1} \\
\mathrm{Fe}^{3+}+\mathrm{H}_{2} \mathrm{O}_{2} \rightarrow \mathrm{Fe}^{2+}+\mathrm{HO}_{2}^{\bullet}+\mathrm{H}^{+} & k=1 \times 10^{-2} \mathrm{~L} \mathrm{~mol}^{-1} \mathrm{~s}^{-1}
\end{array}
$$

Para aumentar a produção de radicais livres por meio da reação do tipo Fenton (a partir de $\mathrm{Fe}^{3+}$ e $\mathrm{H}_{2} \mathrm{O}_{2}$ ), o uso de compostos fenólicos redutores de ferro (CFRF) tem proporcionado melhores resultados, pois os mesmos atuam como mediadores da reação, promovendo a redução contínua de $\mathrm{Fe}^{3+}$ a $\mathrm{Fe}^{2+}$ e obtendo-se assim uma maior geração de ${ }^{\bullet} \mathrm{OH}$ (Chen et al., 2002; Aguiar et al., 2007). Entre os CFRF, destaca-se o grupo de compostos orgânicos classificados como metoxifenois (MFs), os quais se encontram na natureza, principalmente por meio da biodegradação e solubilização de lignina, uma macromolécula aromática que confere resistência às plantas (Aguiar e Ferraz, 2011). Extratos aquosos a partir da biodegradação de madeira apresentam compostos do tipo MF e muitos deles exibem atividade redutora de $\mathrm{Fe}^{3+}$ (Ferraz et al., 2001; Aguiar e Ferraz, 2007). Dentre os MFs, o presente trabalho avaliou o uso do siringaldeído (3,5-dimetoxi,4-hidroxibenzaldeído) para mediar o processo de descoloração de quatro diferentes corantes por reagentes Fenton.

\section{MATERIAIS E MÉTODOS}

Os experimentos de descoloração foram conduzidos em cubetas de quartzo de $3 \mathrm{~mL}$

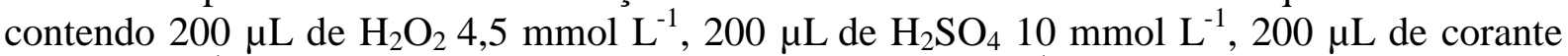
$300 \mu \mathrm{mol} \mathrm{L}{ }^{-1}$ e $200 \mu \mathrm{L}$ de $\mathrm{Fe}\left(\mathrm{NO}_{3}\right)_{3}$ ou de $\mathrm{FeSO}_{4} 300 \mu \mathrm{mol} \mathrm{L}{ }^{-1}$ para as reações tipo Fenton ou Fenton clássica, respectivamente. Quando realizados os experimentos na presença do siringaldeído, $200 \mu \mathrm{L}$ de solução $100 \mu \mathrm{mol} \mathrm{L}^{-1}$ de tal composto foram adicionados. Avolumou-se a cubeta para $2 \mathrm{~mL}$, sendo mantida ao abrigo da luz (Aguiar e Ferraz, 2007). Leituras de descoloração foram realizadas em um espectrofotômetro no comprimento de onda referente à máxima absorção de cada corante $(\lambda=513 \mathrm{~nm}$ - cromotrope $2 \mathrm{R} ; \lambda=508 \mathrm{~nm}$ alaranjado de metila; $\lambda=435 \mathrm{~nm}$ - vermelho de fenol; $\lambda=663 \mathrm{~nm}$ - azul de metileno). A degradação dos corantes foi monitorada por meio da descoloração das soluções em intervalos de 5, 10, 20, 40 e 60 minutos de reação. Preparou-se o branco com água deionizada. Os ensaios foram realizados em duplicata e à temperatura ambiente. Reações de descoloração na ausência dos corantes foram também realizadas. Por meio destas últimas objetivou-se investigar a possível interferência dos produtos de degradação do siringaldeído no processo de descoloração dos corantes em estudo. $\mathrm{O} \mathrm{H}_{2} \mathrm{O}_{2}$ residual foi quantificado com $\mathrm{NH}_{4} \mathrm{VO}_{3}$ em 450 nm (Aguiar e Ferraz, 2007).

\section{RESULTADOS E DISCUSSÃO}

Os ensaios de descoloração dos corantes foram realizados em pH baixo (2,5-3,0), pois diante dessa condição tem-se a solubilidade de sais de ferro aumentada e, principalmente, a 
inibição da precipitação de hidr(óxidos) de ferro (Aguiar et al., 2007). Para os ensaios realizados na presença do siringaldeído, ressalta-se que o ideal é utilizar uma pequena quantidade do mesmo, pois do contrário pode ser observado efeito inibitório devido ao sequestro dos radicais livres pelo siringaldeído em excesso no meio reacional. Esse é um aspecto favorável ao processo, pois é necessária uma quantidade mínima do MF nessas reações e é importante salientar também que a adição deste não deve ser considerada um problema de contaminação adicional, visto que o mediador pode ser degradado durante o tratamento (Pracht et al., 2001). Nos testes realizados na presença de $10 \%$ (v/v) de etanol, o qual é um agente sequestrador de ${ }^{\bullet} \mathrm{OH}$, a descoloração dos corantes em estudo não ocorreu, demonstrando que tais radicais são os principais agentes oxidantes responsáveis pela descoloração dos corantes (Aguiar e Ferraz, 2007). Adicionalmente, também não houve a descoloração dos corantes por apenas $\mathrm{Fe}^{2+}, \mathrm{Fe}^{3+}$ ou $\mathrm{H}_{2} \mathrm{O}_{2}$.

Por meio de ensaios realizados na ausência dos corantes em estudo, foi possível observar que os produtos de degradação do siringaldeído não causaram surgimento de coloração no meio após 60 minutos de reação, ou seja, não interferiram no processo de descoloração. Embora somente a descoloração das soluções contendo os corantes tenha sido analisada neste trabalho, a literatura mostra que os grupos cromóforos são primeiramente atacados e em seguida a molécula de corante alterada é mineralizada por reagentes Fenton, (Xu et al., 2004; Magureanu et al., 2007). Na Figura 1 estão mostradas as cinéticas de descoloração dos corantes por reagentes Fenton. A descoloração por reação de Fenton clássica (apenas $\mathrm{Fe}^{2+} / \mathrm{H}_{2} \mathrm{O}_{2}$ ) foi rápida nos 5 primeiros minutos, tornando-se mais lenta depois, podendo ser dividida em dois estágios: o primeiro corresponde àquele no qual a formação de ${ }^{\bullet} \mathrm{OH}$ ocorre rapidamente e, portanto, a descoloração é mais intensa e o segundo refere-se àquele no qual, devido à menor formação de radicais hidroxila e início da formação de radicas hidroperoxila, tem-se uma descoloração mais lenta (Du et al., 2006). Para o sistema $\mathrm{Fe}^{2+} / \mathrm{H}_{2} \mathrm{O}_{2} /$ siringaldeído, observou-se que antes dos 60 min houve inibição na descoloração, a qual deve estar associada às propriedades antioxidantes do mediador ao competir com os corantes por ${ }^{\bullet} \mathrm{OH}$ (Moran et al., 1997; Briante et al., 2003; Antolovich et al., 2004). Após 60 min de reação, é possível observar uma tendência de aumento da descoloração dos corantes por siringaldeído, devido à provável regeneração de íons $\mathrm{Fe}^{2+}$.

Os sistemas reacionais contendo apenas $\mathrm{Fe}^{3+} / \mathrm{H}_{2} \mathrm{O}_{2}$ praticamente não promoveram a descoloração do alaranjado de metila e do vermelho de fenol, mas apenas do cromotrope $2 \mathrm{R}$ e do azul de metileno, os quais atingiram $27 \%$ e $31 \%$ de descoloração após 60 minutos de reação, respectivamente. Sabe-se que na reação tipo Fenton, a espécie $\mathrm{Fe}^{2+}$ é gerada lentamente através da redução de $\mathrm{Fe}^{3+}$ por $\mathrm{H}_{2} \mathrm{O}_{2}$, conforme a Equação 2 e então a reação de Fenton clássica é iniciada. Porém, ao mesmo tempo, $\mathrm{Fe}^{2+}$ é rapidamente re-oxidado a $\mathrm{Fe}^{3+}$, o que justifica o motivo pelo qual a descoloração dos corantes por reação tipo Fenton é bem mais lenta que a clássica. Na presença do siringaldeído, $69 \%$ e $59 \%$ de descoloração por $\mathrm{Fe}^{3+} / \mathrm{H}_{2} \mathrm{O}_{2}$ para os corantes cromotrope $2 \mathrm{R}$ e azul de metileno foram observados após 60 minutos de reação, respectivamente. Este fato demonstra o efeito pró-oxidante do mediador natural em estudo, sendo que tais porcentagens são próximas àquelas obtidas para a reação de Fenton clássica. Entretanto, para o alaranjado de metila e vermelho de fenol, corantes mais recalcitrantes diante dos tratamentos de descoloração em estudo, o siringaldeído não apresentou efeito pró-oxidante durante os 60 min de reação usando íons $\mathrm{Fe}^{3+}$. 

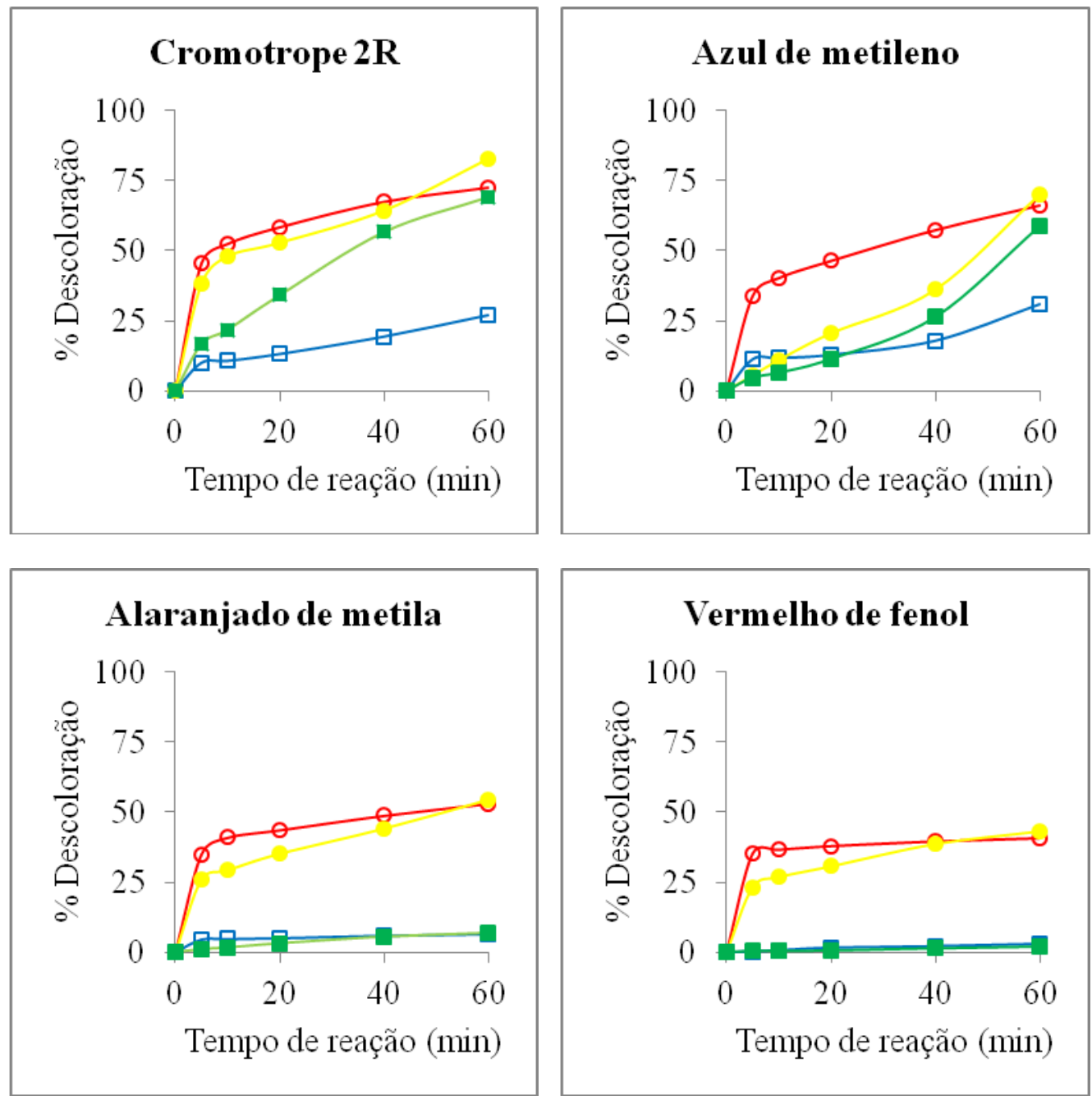

Figura 1 - Curvas de descoloração dos corantes cromotrope 2R, azul de metileno, alaranjado de metila e vermelho de fenol, todos na concentração de $30 \mu \mathrm{mol} \mathrm{L}{ }^{-1} \mathrm{em} \mathrm{pH}$ 2,8, promovidas por: $30 \mu \mathrm{mol} \mathrm{L}{ }^{-1} \mathrm{de} \mathrm{Fe}^{3+}$ e $450 \mu \mathrm{mol} \mathrm{L}^{-1} \mathrm{de}_{2} \mathrm{O}_{2}(\square) ; 30 \mu \mathrm{mol} \mathrm{L}{ }^{-1}$ de $\mathrm{Fe}^{2+}$ e $450 \mu \mathrm{mol} \mathrm{L}{ }^{-1}$ de $\mathrm{H}_{2} \mathrm{O}_{2}(\mathrm{O}) ; 30 \mu \mathrm{mol} \mathrm{L}^{-1}$ de Fe${ }^{3+}, 450 \mu \mathrm{mol} \mathrm{L}^{-1}$ de $\mathrm{H}_{2} \mathrm{O}_{2}$ e $10 \mu \mathrm{mol}$ $\mathrm{L}^{-1}$ de siringaldeído ( $\square$ ) e $30 \mu \mathrm{mol} \mathrm{L}{ }^{-1} \mathrm{de} \mathrm{Fe}^{2+}, 450 \mu \mathrm{mol} \mathrm{L}^{-1}$ de $\mathrm{H}_{2} \mathrm{O}_{2}$ e $10 \mu \mathrm{mol} \mathrm{L}{ }^{-1}$ de siringaldeído ( $)$. Os experimentos de descoloração foram realizados em duplicata e apresentaram erros inferiores a 5\% quando comparados ao valor médio.

Os distintos comportamentos de descoloração entre os corantes investigados neste trabalho podem ser atribuídos à diferente reatividade de cada grupo cromóforo e outros sítios reacionais presentes nas moléculas dos mesmos. O carbono metino central no vermelho de fenol é um importante local de ataque por ${ }^{\bullet} \mathrm{OH}$, levando a sua descoloração e clivagem e os anéis aromáticos podem ser hidroxilados ou clivados (Abdelmalek et al., 2006; Magureanu et al., 2007). A descoloração do azul de metileno se inicia com a clivagem do grupo funcional $\mathrm{C}-\mathrm{S}^{+}=\mathrm{C}$, ao passo que a oxidação do cromotrope $2 \mathrm{R}$ procede pela adição de um ${ }^{\bullet} \mathrm{OH}$ ao átomo de carbono que suporta a ligação $-\mathrm{N}=\mathrm{N}-$ (Lachheb et al., 2002). No entanto, o substituinte $-\mathrm{N}\left(\mathrm{CH}_{3}\right)_{2}$, grupo não cromóforo presente no azul de metileno e no alaranjado de metila, também é um importante sítio de ataque por ${ }^{\bullet} \mathrm{OH}$ (Lachheb et al., 2002; Abdelmalek et al., 2006; Magureanu et al., 2007; Devi et al., 2010). Corroborando as diferentes 
reatividades dos corantes no presente trabalho, Xu et al. (2004) investigaram a descoloração de vinte corantes, incluindo os dos grupos azo, trifenilmetileno, antraquinona, heterocíclicos e ftalocianina e eles não observaram uma descoloração mais efetiva por sistemas Fenton para um grupo específico de corantes.

A Tabela 1 mostra os dados de consumo de $\mathrm{H}_{2} \mathrm{O}_{2}$ para as reações de descoloração após 60 minutos e também para as reações sem corantes (controle). Comparando a reação de Fenton clássica com a reação tipo Fenton para os diversos sistemas reacionais, a primeira apresentou maior consumo de $\mathrm{H}_{2} \mathrm{O}_{2}$, provavelmente devido à sua mais alta constante cinética de reação. Descoloração por $\mathrm{Fe}^{2+} / \mathrm{H}_{2} \mathrm{O}_{2}$ /siringaldeído também consumiu mais $\mathrm{H}_{2} \mathrm{O}_{2}$ que $\mathrm{Fe}^{3+} / \mathrm{H}_{2} \mathrm{O}_{2}$ /siringaldeído. Em geral, as reações com o cromotrope $2 \mathrm{R}$, corante que mostrou-se mais passível aos tratamentos de descoloração em estudo, e as reações na ausência de corantes consumiram mais e menos $\mathrm{H}_{2} \mathrm{O}_{2}$, respectivamente. A partir desta última observação, pode-se inferir que intermediários gerados no processo de descoloração dos corantes podem afetar a conversão $\mathrm{Fe}^{3+} / \mathrm{Fe}^{2+}$ e consequentemente aumentar o consumo de $\mathrm{H}_{2} \mathrm{O}_{2}$ quando comparado com as reações sem corantes.

Apesar dos sistemas reacionais contendo $\mathrm{Fe}^{3+}$ não promoverem a descoloração dos corantes vermelho de fenol e alaranjado de metila, o consumo de $\mathrm{H}_{2} \mathrm{O}_{2}$ foi significativo. Estes resultados sugerem que radicais livres resultantes do consumo de $\mathrm{H}_{2} \mathrm{O}_{2}$ por $\mathrm{Fe}^{3+} \mathrm{e}$ $\mathrm{Fe}^{3+} /$ siringaldeído aparentemente não possuem potencial padrão de redução suficiente para descolorir esses corantes. Para o cromotrope $2 \mathrm{R}$ e o azul de metileno, ainda que $\mathrm{o}$ siringaldeído tenha elevado consideravelmente as porcentagens de descoloração, ele diminuiu o consumo de $\mathrm{H}_{2} \mathrm{O}_{2}$ para o primeiro corante, enquanto para o segundo houve apenas um pequeno aumento no consumo de $\mathrm{H}_{2} \mathrm{O}_{2}$.

Tabela $1-\mathrm{H}_{2} \mathrm{O}_{2}$ consumido (\%) após 60 minutos de reação para descoloração dos corantes por reagentes Fenton na presença ou ausência do siringaldeído. Os desvios dos valores médios são baseados em experimentos realizados em duplicata

\begin{tabular}{|c|c|c|c|c|c|}
\hline \multirow{2}{*}{ Sistema reacional } & \multicolumn{5}{|c|}{$\% \mathrm{H}_{2} \mathrm{O}_{2}$ consumido } \\
\cline { 2 - 6 } & $\begin{array}{c}\text { Cromotrope } \\
2 \mathrm{R}\end{array}$ & $\begin{array}{c}\text { Azul de } \\
\text { metileno }\end{array}$ & $\begin{array}{c}\text { Alaranjado } \\
\text { de metila }\end{array}$ & $\begin{array}{c}\text { Vermelho } \\
\text { de Fenol }\end{array}$ & $\begin{array}{c}\text { Ausência de } \\
\text { corante }\end{array}$ \\
\hline $\mathrm{Fe}^{3+} / \mathrm{H}_{2} \mathrm{O}_{2}$ & $69,2 \pm 0,3$ & $58,5 \pm 0,6$ & $52,1 \pm 2,0$ & $50,7 \pm 1,0$ & $32,1 \pm 1,5$ \\
\hline $\mathrm{Fe}^{2+} / \mathrm{H}_{2} \mathrm{O}_{2}$ & $77,6 \pm 1,9$ & $72,5 \pm 1,6$ & $62,8 \pm 1,2$ & $59,1 \pm 1,5$ & $48,3 \pm 0,1$ \\
\hline $\mathrm{Fe}^{3+} / \mathrm{H}_{2} \mathrm{O}_{2} /$ siringaldeído & $64,6 \pm 0,6$ & $61,4 \pm 0,3$ & $60,5 \pm 1,9$ & $51,1 \pm 1,6$ & $43,4 \pm 0,6$ \\
\hline $\mathrm{Fe}^{2+} / \mathrm{H}_{2} \mathrm{O}_{2} /$ siringaldeído & $72,3 \pm 0,9$ & $65,5 \pm 0,0$ & $67,3 \pm 1,8$ & $56,8 \pm 0,3$ & $48,2 \pm 0,0$ \\
\hline
\end{tabular}

Elevados consumos de $\mathrm{H}_{2} \mathrm{O}_{2}$ associados aos sistemas nos quais não foram observadas porcentagens de descoloração significativas, podem ser também justificados pelo fato que, apesar dos radicais hidroxila terem sido formados eles não são seletivos, ou seja, os mesmos podem ter reagido com o siringaldeído ou ainda com o seu próprio precursor $\left(\mathrm{H}_{2} \mathrm{O}_{2}\right)$ ao invés de atuarem no processo de descoloração dos corantes. Nesse segundo caso, conforme a reação representada pela Equação 3, tem-se a geração do radical hidroperoxila, o qual possui menor potencial padrão de redução que o radical hidroxila. 


$$
{ }^{\bullet} \mathrm{OH}+\mathrm{H}_{2} \mathrm{O}_{2} \rightarrow \mathrm{H}_{2} \mathrm{O}+\mathrm{HO}_{2}^{\bullet}
$$

\section{CONCLUSÃO}

Através do presente trabalho, avaliou-se a descoloração de quatro diferentes corantes em solução aquosa por sistemas Fenton na presença e ausência do mediador siringaldeído. Notou-se que a adição deste metoxifenol pode aumentar efetivamente a reatividade de oxidação no processo de descoloração de corantes menos recalcitrantes, ou seja, o mesmo apresentou propriedades pró-oxidantes na reação tipo Fenton, representando uma abordagem promissora ao tratamento ambientalmente amigável de águas residuais. Diferentes comportamentos de descoloração e de consumo do $\mathrm{H}_{2} \mathrm{O}_{2}$ para os quatro corantes testados foram observados, sugerindo que a estrutura dos mesmos tem influência nas reações.

\section{AGRADECIMENTOS}

Os autores agradecem a Fundação de Amparo à Pesquisa do estado de Minas Gerais (FAPEMIG, processo APQ-01585-11) pela concessão de auxílio financeiro.

\section{REFERÊNCIAS BIBLIOGRÁFICAS}

ABDELMALEK, F.; GHEZZAR, M. R.; BELHADJ, M.; ADDOU, A.; BRISSET J. L. Bleaching and degradation of textile dyes by nonthermal plasma process at atmospheric pressure. Ind. \& Eng.Chem. Res., v. 45, p. 23-29, 2006.

AGUIAR, A.; FERRAZ, A. Effect of aqueous extracts from Ceriporiopsis subvermisporabiotreated wood on the decolorization of Azure B by Fenton-like reactions. Int. Biodet. Biodegr., v. 74, p. 61-66, 2012.

AGUIAR, A.; FERRAZ, A. Mecanismos envolvidos na biodegradação de materiais lignocelulósicos e aplicações tecnológicas correlatas. Quim. Nova, v. 34, p. 1729-1738, 2011.

AGUIAR, A.; FERRAZ, A. $\mathrm{Fe}^{3+}$ - and $\mathrm{Cu}^{2+}$ - reduction by phenol derivatives associated with Azure B degradation in Fenton-like reactions. Chemosphere, v. 66, p. 947-954, 2007.

AGUIAR, A.; FERRAZ, A.; CONTRERAS, D.; RODRÍGUEZ, J. Mecanismo e aplicações da reação de Fenton assistida por compostos fenólicos. Quim. Nova, v. 30, p. 623-628, 2007.

ANTOLOVICH, M.; BEDGOOD JR., D. R.; BISHOP, A. G.; JARDINE, D.; PRENZLER, P. D.; ROBARDS, K. LC-MS investigation of oxidation products of phenolic antioxidants. J. Agric. Food Chem., v. 52, p. 962-971, 2004. 
BRIANTE, R.; FEBBRAIO, F.; NUCCI, R. Antioxidant properties of low molecular weight phenols present in the Mediterranean diet. J. Agric. Food Chem., v. 51, p. 6975-6981, 2003.

CHEN, F.; MA, W. H.; HE, J. J.; ZHAO, J. C. Fenton degradation of malachite green catalyzed by aromatic additives. J. Phys. Chem., v. 106, p. 9485-9490, 2002.

DEVI, L. G.; KUMAR, S. G.; RAJU, K. S. A.; RAJASHEKHAR, K. E. Photo-Fenton and photo-Fenton-like processes for the degradation of methyl orange in aqueous medium: influence of oxidation states of iron. Chem. Pap., v. 64, p. 378-385, 2010.

DU, Y.; ZHOU M.; LEI L. Role of the intermediates in the degradation of phenolic compounds by Fenton-like process. J. Hazard. Mat., v. 136, p. 859-865, 2006.

FERRAZ, A.; PARRA, C.; FREER, J.; BAEZA, J.; RODRÍGUEZ, J. Occurrence of ironreducing compounds in biodelignified "palo podrido" wood samples. Int. Biodet. Biodegr., v. 47, p. 203-208, 2001.

KAUSHIK, P.; MALIK A. Fungal decolorization: Recent advances and future potential. Environ. Intern., v. 35, p. 127-141, 2009.

LACHHEB, H.; PUZENAT, E.; HOUAS, A.; KSIBI, M.; ELALOUI, E.; GUILLARD, C.; HERRMANN, J. M. Photocatalytic degradation of various types of dyes (Alizarin S, Crocein Orange G, Methyl Red, Congo Red, Methylene Blue) in water by UVirradiated titania. Appl. Catal. B: Environ., v. 39, p. 75-90, 2002.

MA, J.; MA, W.; SONG, W.; CHEN, C.; TANG, Y.; ZHAO, J.; HUANG, Y.; XU, Y.; ZANG, L. Fenton degradation of organic pollutants in the presence of low-molecularweight organic acids: cooperative effect of quinone and visible light. Environ. Sci. Technol., v. 40, p. 618-624, 2006.

MAGUREANU, M.; MANDACHE, N. B.; PARVULESCU, V. I. Degradation of organic dyes in water by electrical discharges. Plasma Chem. Plasma Process., v. 27, p. 589598, 2007.

MORAN, J. F.; KLUCAS, R. V.; GRAYER, R. J.; ABIAN, J.; BECANA, M. Complexes of iron with phenolic compounds from soybean nodules and other legume tissues: prooxidant and antioxidant properties. Free Radical Biol. Med., v. 22, p. 861-870, 1997.

PRACHT, J.; BOENIGK, J.; ISENBECK-SCHOTER, M.; KEPPLER, F.; SCHOLER, H.F. Abiotic Fe(III) induced mineralization of phenolic substances. Chemosphere, v. 44, p. 613-619, 2001.

XU, X. R.; LI H. B.; WANG, W. H.; GU, J. D. Degradation of dyes in aqueous solutions by the Fenton process. Chemosphere, v. 5, p. 595-600, 2004. 